\title{
Clinical outcomes and safety of rituximab treatment for patients with systemic lupus erythematosus (SLE) - results from a nationwide cohort in Germany (GRAID)
}

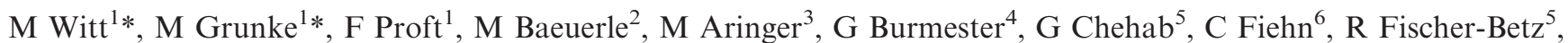
M Fleck ${ }^{7}, \mathrm{~K}_{\text {Freivogel }}{ }^{8}$, M Haubitz ${ }^{9}$ I Kötter ${ }^{10}$, S Lovric ${ }^{11}$, C Metzler ${ }^{12}$, A Rubberth-Roth ${ }^{13}$, A Schwarting ${ }^{14}$, C Specker ${ }^{15}$, H-P Tony ${ }^{16}$, L Unger ${ }^{17}$, S Wassenberg ${ }^{18}$, T Dörner ${ }^{4}$ and H Schulze-Koops ${ }^{1}$ on behalf of the German Registry of Autoimmune Diseases (GRAID) Investigators ${ }^{1}$ Division of Rheumatology, Medizinische Klinik und Poliklinik IV, University Hospital Munich, Germany; ${ }^{2}$ Rheumatologie, Klinikum Nuernberg, Germany; ${ }^{3}$ Division of Rheumatology, Department of Medicine III, University Clinical Center Carl Gustav Carus, Germany; ${ }^{4}$ Rheumatology and Clinical Immunology, Charité University Hospital, Germany; ${ }^{5}$ Clinic for Rheumatology, Heinrich-Heine-University, Germany; ${ }^{6}$ Center for Rheumatic Diseases, Germany; ${ }^{7}$ Department of Internal Medicine I, University of Regensburg, Germany; ${ }^{8}$ Analytica International GmbH, Germany; ${ }^{9}$ Department of Internal Medicine III, Klinikum Fulda, Germany; ${ }^{10}$ Rheumatology Outpatient Clinic, Germany;

${ }^{11}$ Department of Nephrology, Hannover Medical School, Germany; ${ }^{12}$ Internal Medicine II, Barmherzige Brueder Hospital, Germany; ${ }^{13}$ Department of Rheumatology, University Hospital of Cologne, Germany; ${ }^{14}$ Division of Rheumatology and Clinical Immunology, Dept. of Internal Medicine, Johannes Gutenberg-Universität, Germany; ${ }^{15}$ Department of Rheumatology and Clinical Immunology, Kliniken Essen-Sued, Germany; ${ }^{16}$ Medizinische Klinik and Poliklinik II, University of Wuerzburg, Germany; ${ }^{17}$ Department of Rheumatology, Internal Medicine I, Krankenhaus Dresden-Friedrichstadt, Germany; and ${ }^{18}$ Department of Rheumatology, Evangelisches Fachkrankenhaus Ratingen, Germany
\end{abstract}

\begin{abstract}
Objective: The objective of this article is to evaluate the safety and clinical outcome of rituximab treatment in systemic lupus erythematosus (SLE) patients refractory to standard of care therapy in a real-life setting in Germany. Methods: The GRAID registry included patients with different autoimmune diseases who were given off-label treatment with rituximab. Data on safety and clinical response were collected retrospectively. In SLE patients, clinical parameters included tender and swollen joint counts, fatigue, myalgia, general wellbeing, Raynaud's and the SLEDAI index. Laboratory tests included dsDNA antibody titres, complement factors, hematologic parameters and proteinuria. Finally, the investigators rated their patients as non-, partial or complete responders based on clinical grounds. Results: Data from 85 SLE patients were collected, 69 female and 16 male, with a mean disease duration of 9.8 years. The mean follow-up period was $9.6 \pm 7.4$ months, resulting in 66.8 patient years of observation. A complete response was reported in 37 patients $(46.8 \%)$, partial response in 27 $(34.2 \%)$, no response in $15(19.0 \%)$. On average, major clinical as well as laboratory efficacy parameters improved substantially, with the SLEDAI decreasing significantly from 12.2 to 3.3 points. Concerning safety, one infusion reaction leading to discontinuation of treatment occurred. Infections were reported with a rate of 19.5 (including six severe infections) per 100 patient years. Conclusion: With the restrictions of a retrospective data collection, the results of this study confirm data of other registries, which suggest a favourable benefit-risk ratio of rituximab in patients with treatment-refractory SLE. Lupus (2013) 22, 1142-1149.
\end{abstract}

Key words: Systemic lupus erythematosus; rituximab; efficacy; safety; B lymphocytes; cohort studies

\section{Introduction}

The role of B cells in the pathogenesis of systemic lupus erythematosus (SLE) and other systemic

Correspondence to: Matthias Witt, Med. Klinik und Poliklinik IV, Klinikum der Universität München, Pettenkoferstr. 8a, 80336 München, Germany.

Email: Matthias.Witt@med.uni-muenchen.de

*M.W. and M.G. contributed equally to this work.

Received 5 April 2013; accepted 9 August 2013 autoimmune diseases is widely acknowledged. ${ }^{1}$ It is not only their function as precursors to antibody-producing cells, but also their ability to present antigen and to activate $T$ cells as well as their ability to secrete various pro-inflammatory cytokines, which makes them a promising target in autoimmune disorders such as SLE., ${ }^{2,3}$ The theoretical concept is supported by a large body of reports that describe a successful intervention in a number of autoimmune diseases by either B cell depletion or modulation. ${ }^{4}$ 
Rituximab is a B cell-depleting anti-CD20 antibody which has recently been approved as first-line therapy for antineutrophil cytoplasmic antibody (ANCA)-associated small vessel vasculitides (AAV) by the United States Food and Drug Administration (FDA) and the European Medical Agency (EMA), making AAV the third indication for rituximab besides lymphoma and rheumatoid arthritis (RA). ${ }^{5,6}$ In addition, rituximab has been used for off-label treatment of refractory SLE for more than 10 years. ${ }^{7-9}$ However, two recent randomized controlled trials with rituximab in SLE (EXPLORER) and lupus nephritis (LUNAR) did not reach their primary efficacy endpoints. ${ }^{10,11}$ There has been much debate about the reasons for this lack of evidence and concerns about the design of these trials as clinical experience of many experts in the field and results of large open-label studies favour the use of rituximab in this disease. ${ }^{12-16}$

Although further clinical trials may be launched to further investigate the efficacy of rituximab in SLE, it is unlikely that approval of rituximab for SLE will be gained in the near future. Given the urgent need for effective, safe and tolerable treatment options for SLE patients, in particular in disease refractory to standard measures of care, it is of great importance that experiences with the off-label use of rituximab are collected and made accessible to physicians treating these patients. ${ }^{17}$

The German Registry of Autoimmune Diseases (GRAID) has been established to provide further evidence on the safety and clinical outcomes of rituximab in patients with different autoimmune diseases on a retrospective basis. It includes not exclusively rheumatologic, but also dermatologic, neurologic and nephrologic cases which have been refractory to standard of care treatment and therefore have been treated with off-label rituximab. An overview of the observations in the registry has been published recently. ${ }^{18}$ This manuscript focuses on the data of the SLE patients, which represented the largest population within GRAID.

\section{Patients and methods}

The design of this trial was a multicentre, noninterventional retrospective data collection of adult patients with different autoimmune disorders, who had received rituximab on an off-label basis as deemed necessary by their treating physicians. General reasons for rituximab treatment in this cohort were inadequate response to, side effects of and/or contraindications for the preceding
SLE treatments. Inefficacy was defined as recurrent and/or persistent SLE symptoms according to the treating physician despite combination therapy with glucocorticoids and disease-modifying antirheumatic drugs (DMARDs) or cyclophosphamide. Patients with diagnoses approved for treatment with rituximab, e.g. RA or nonHodgkin's lymphoma, were not included. Rituximab was either added to or replaced the concurrent immunosuppressive therapy. The diagnosis of SLE and its clinical manifestations such as serositis and anaemia, etc., were based on the judgement of the treating physicians, who were requested to include only patients fulfilling the current American College of Rheumatology (ACR) criteria for SLE. ${ }^{19}$ Lupus nephritis was included if results of renal biopsies were consistent with the diagnosis. Safety of Estrogens in Lupus Erythematosus National Assessment-Systemic Lupus Erythematosus Disease Activity Index (SELENA-SLEDAI) scores at baseline and at last follow-up were calculated as specified previously if appropriate data were available. ${ }^{20}$ All laboratory tests such as assessment of complement levels and anti-double-stranded DNA (anti-dsDNA) antibodies were performed at the sites.

Because of the diversity of included diagnoses and the retrospective nature of the survey, efficacy assessments were restricted to a categorization of complete response, partial response and no response, as judged at the discretion of the treating physician. Additionally, the investigators estimated the patients' wellbeing on a $100 \mathrm{~mm}$ visual analogue scale (VAS). Response of rituximab in SLE patients was further evaluated by comparison of mean SELENA-SLEDAI scores at baseline and after last infusion. Other instruments of efficacy assessment included the percentage of patients with normalization of complement and dsDNA antibody levels and the proportion of patients with corticosteroid reduction.

Safety assessments included the registration of adverse events (AE) and serious AEs during the treatment and follow-up period as well as all adverse reactions that occurred during or within 24 hours of rituximab infusions. The intensity of AEs was graded using the National Cancer Institute (NCI) Common Terminology Criteria for Adverse Events (CTCAE), version 3 or a grade $1-5$ severity scale. ${ }^{21}$ Infections were classified as serious if they led to intravenous (i.v.) antibiotic treatment, hospitalization or death. The specification of the severity of infections as mild, moderate and severe was based on the treating investigators' estimation. 
Data entry for GRAID occurred via Web-based electronic case report forms between December 2008 and July 2009. Participating physicians came from 42 hospital and private practice centres in Germany and were not only rheumatologists, but also haematologists, nephrologists, neurologists, dermatologists and other internal medicine specialists as well.

Ethical approval and approval by the local data protection agency were obtained by Charité Universitaetsmedizin Berlin. Local approval was obtained by the principal investigators if required by local regulations. Statistical analysis was performed with Prism 5 software for Mac OS X using two-tailed $t$ - and Chi-square tests. $P$ values of less than 0.05 were considered significant.

\section{Results}

\section{Baseline and treatment characteristics}

Among the 370 patients reported to the GRAID registry, $85(23.0 \%)$ had a diagnosis of SLE, representing the most common GRAID disorder. Sixtynine $(81.0 \%)$ SLE patients were female. The mean disease duration was $9.8 \pm 8$ years. Before rituximab treatment, the most common SLE manifestations in the cohort were fatigue $(48.2 \%)$, erythema (42.4\%), anaemia (38.8\%), myalgia $(34.1 \%)$ and arthritis (29.4\%). In addition, 36.5\% of the patients had biopsy-proven lupus nephritis.

Reasons for the use of rituximab in this cohort were inefficacy, side effects and/or contraindications to preceding treatments in $71(89.9 \%), 25$ $(31.6 \%)$ and $11(13.9 \%)$ patients, respectively. Independent side effects included nausea, bone marrow toxicity and renal insufficiency, among others. Advanced renal insufficiency with a glomerular filtration rate (GFR) below $30 \mathrm{ml} / \mathrm{min}$ is an example of a contraindication to non-rituximab treatments. In the group of patients with inadequate response to the preceding treatments, the indications for rituximab were renal involvement, i.e. glomerulonephritis and/or proteinuria, in 27 patients, haematologic manifestations, i.e. anaemia, leucopenia and/or thrombocytopenia, in 21 patients, central nervous system involvement in seven patients, skin involvement in six patients, serositis in three patients and other reasons, e.g. arthritis, in 15 patients. In two patients, non-compliance with conventional treatment was the reason for the use of rituximab.

Prior to the first course of rituximab, all patients had been treated with corticosteroids, $41.8 \%$ with mycophenolate mofetil, $39.2 \%$ with azathioprine, $34.2 \%$ with antimalarials, $32.9 \%$ with cyclophosphamide, $20.2 \%$ with methotrexate, $11.4 \%$ with i.v. immunoglobulins (IvIgs) and $8.9 \%$ with leflunomide. During rituximab therapy, concomitant treatment consisted of corticosteroids in $92.4 \%$, mycophenolate mofetil in $30.4 \%$, antimalarials in $29.1 \%$, azathioprine in $11.4 \%$, cyclophosphamide in $7.6 \%$, methotrexate in $3.8 \%$, IvIg in $3.8 \%$ and others in $2.5 \%$. An overview of the baseline characteristics of the SLE population is shown in Table 1.

Sixty-seven (78.8\%), $16(18.8 \%)$ and two $(2.4 \%)$ of the patients received one, two and three treatment courses, respectively. A treatment course was defined as two infusions of rituximab in a two-week interval. The mean interval between two treatment

Table 1 Overview of the baseline characteristics of the GRAID SLE cohort

\begin{tabular}{|c|c|}
\hline \multicolumn{2}{|l|}{ Epidemiology } \\
\hline Patients treated, $n$ & 85 \\
\hline Female $(\%)$ & $81.0 \%$ \\
\hline age at first infusion ${ }^{\mathrm{a}}$ (years) & 36.6 \\
\hline disease duration $^{\mathrm{a}}$ (years with SD) & $9.8 \pm 8.0$ \\
\hline \multicolumn{2}{|l|}{ Key clinical features } \\
\hline Fatigue $(\%)$ & 48.2 \\
\hline Erythema $(\%)$ & 42.4 \\
\hline Myalgia (\%) & 34.1 \\
\hline Arthritis $(\%)$ & 29.4 \\
\hline Lupus nephritis $(\%)$ & 36.7 \\
\hline SELENA-SLEDAI score ${ }^{\mathrm{a}}$ & $12.2 \pm 7.4$ \\
\hline \multicolumn{2}{|l|}{ Biomarkers } \\
\hline $\operatorname{ESR}^{\mathrm{a}}(\mathrm{mm} / \mathrm{h})$ & $36.0 \pm 25.3$ \\
\hline $\mathrm{C} 3^{\mathrm{a}}(\mathrm{mg} / \mathrm{dl})$ & $68.3 \pm 36.6$ \\
\hline $\mathrm{C}^{\mathrm{a}}(\mathrm{mg} / \mathrm{dl})$ & $10.8 \pm 9.1$ \\
\hline Elevated ANA titre $(\%)$ & 84.1 \\
\hline Elevated dsDNA antibodies (\%) & 73.4 \\
\hline Proteinuria $(\%)$ & 38.8 \\
\hline \multicolumn{2}{|l|}{ Concomitant medications (\%) } \\
\hline Corticosteroids & 92.4 \\
\hline Mycophenolate mofetil & 30.4 \\
\hline Antimalarials & 29.1 \\
\hline Azathioprine & 11.4 \\
\hline Cyclophosphamide & 7.6 \\
\hline Methotrexate & 3.8 \\
\hline IvIg & 3.8 \\
\hline Other & 2.5 \\
\hline
\end{tabular}

GRAID: German Registry of Autoimmune Diseases; SLE: systemic lupus erythematosus; SELENA-SLEDAI: Safety of Estrogens in Lupus Erythematosus National Assessment-Systemic Lupus Erythematosus Disease Activity Index; ESR: erythrocyte sedimentation rate; ANA: antinuclear antibodies; dsDNA: double-stranded DNA; IvIg: intravenous immunoglobulin. ${ }^{a}$ Means with standard deviation (SD); reference values: ESR: $<20 \mathrm{~mm} / \mathrm{h}$ (male), $<30 \mathrm{~mm} / \mathrm{h}$ (female), C3: 90-180 mg/dl, C4: 10-40 mg/dl, dsDNA antibodies positive according to cut-off values of local laboratories, proteinuria $>150 \mathrm{mg} / 24$ hours. 
courses was $13 \pm 9$ months (median 10 months). The mean dosage of rituximab per treatment course was $1887 \pm 670 \mathrm{mg}$ (median $2000 \mathrm{mg}$ ). The mean follow-up period, defined as the time between the first infusion and 180 days after the last infusion, was $9.6 \pm 7.4$ months, summing up to 66.8 patient years of observation. Rituximab treatment was ongoing at the time of data collection in 66 of the 85 patients, while 19 had discontinued rituximab infusions. The reasons were inadequate response in 14, adverse reactions in three, and both reasons in one patient. For another patient the reason for treatment discontinuation was not documented. Details of the rituximab treatment are given in Table 2 .

\section{Efficacy assessment}

The global treatment response as estimated by the treating physicians was complete in $37(46.8 \%)$, partial in $27(34.2 \%)$ and none in $15(19.0 \%)$ of the SLE patients (Figure 1). In addition, the value for general wellbeing as marked on a $100 \mathrm{~mm}$ VAS

Table 2 Details of rituximab treatments in the GRAID SLE cohort

\begin{tabular}{ll}
\hline Courses of rituximab & $\mathrm{n}(\%)$ \\
\hline 1 & $67(78.8)$ \\
2 & $16(18.8)$ \\
3 & $2(2.4)$ \\
Dosage per course (mean mg with SD) & $1887 \pm 670$ \\
Total dosage per patient (mean mg with SD) & $2331 \pm 1033$ \\
Time between two courses (mean months with SD) & $13 \pm 9$ \\
Rituximab discontinued (\%) & $19(22.4)$ \\
Inadequate response & 14 \\
Side effects & 3 \\
Others & 2 \\
\hline
\end{tabular}

GRAID: German Registry of Autoimmune Diseases; SLE: systemic lupus erythematosus; SD: standard deviation.

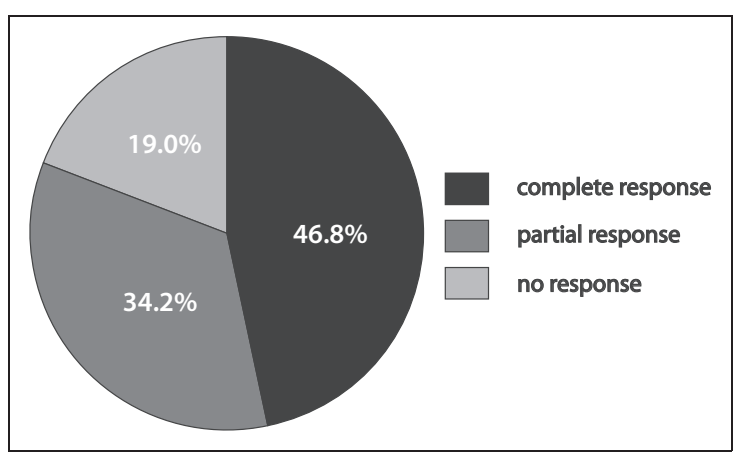

Figure 1 Assessment of treatment response to rituximab according to the physicians' judgement (see Results for details). increased significantly from a mean of 49.2 before the initiation of rituximab treatment to 64.3 at the last visit $(p<0.05)$. Mean SELENA-SLEDAI scores decreased significantly from 12.2 to 3.3 during rituximab treatment $(p<0.05)$. Major disease manifestations such as general, dermatologic and haematologic involvements significantly improved upon rituximab treatment. Specifically, the presence of the following signs and symptoms decreased significantly: the general manifestations fever $(20.0 \%$ to $8.2 \%)$, weight loss $(11.8 \%$ to $2.4 \%$ ), fatigue $(48.2 \%$ to $28.2 \%)$, the presence of the skin symptoms erythema $(42.4 \%$ to $21.2 \%)$, mucocutaneous involvement $(21.2 \%$ to $10.6 \%)$ and Raynaud's syndrome $(35.3 \%$ to $18.8 \%)$, pleuritic symptoms $(12.9 \%$ to $3.5 \%)$, the haematologic manifestations anaemia $(38.8 \%$ to $23.5 \%)$, leucopenia $(20.5 \%$ to $9.1 \%)$ and thrombocytopenia $(32.9 \%$ to $11.8 \%)$, and finally glomerulonephritis $(36.5 \%$ to $21.5 \%)$, with $p<0.05$ each. Other manifestations such as musculoskeletal and neurologic manifestations also improved but did not reach statistical significance (Figure 2). Although the proportion of patients requiring prednisone did not differ significantly before and after rituximab $(86.1 \%$ vs $84.8 \%)$, the necessity for i.v. methylprednisone use could be reduced significantly $(12.7 \%$ vs $1.3 \%, p<0.05)$.

Concerning laboratory markers of activity, $68.1 \%$ and $62.7 \%$ of the patients had low C3 and C4 complement levels at baseline, respectively, compared to $33.3 \%$ and $43.8 \%$ at last visit $(p<0.05$ each). Mean complement C3 and C4 levels increased from 68.3 to $77.5 \mathrm{mg} / \mathrm{dl}$ and from 10.8 to $12.4 \mathrm{mg} / \mathrm{dl}$, respectively (not significant). Further, the proportion of patients with leucopenia was reduced significantly from $20.5 \%$ to $9.1 \%$ $(p<0.05)$. Although not statistically significant, the proportion of patients with elevated dsDNA antibody levels decreased from $73.4 \%$ to $62.2 \%$. The proportion of patients with proteinuria, significant proteinuria and elevated erythrocyte sedimentation rate did not change significantly when pre- and post-rituximab values were compared (Table 3).

\section{Safety assessment}

Adverse reactions occurring during infusions or within 24 hours were reported in 18 of the 85 $(21.2 \%)$ SLE patients. Five of these were defined as allergic reactions $(5.9 \%)$. Only one of the infusion-related reactions resulted in a permanent treatment discontinuation. The overall rate of infections was 19.5 per 100 patient years, including a rate of 


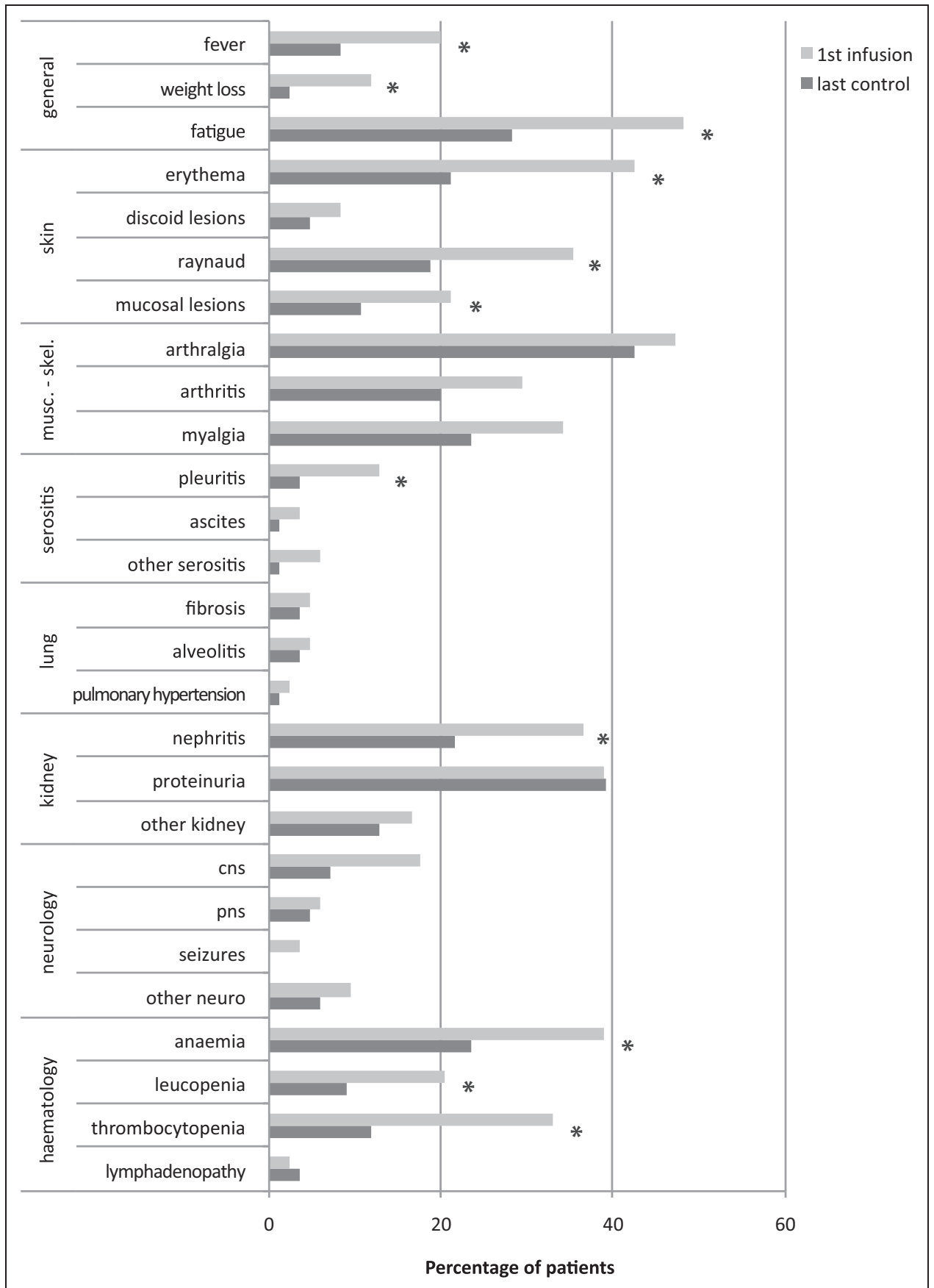

Figure 2 Comparison of the prevalences of lupus manifestations at first infusion of rituximab and at last follow-up. $(*: p<0.05)$. CNS: central nervous system; PNS: peripheral nervous system; musc.-skel.: muskuloskeletal.

severe infections of six per 100 patient years. Twelve of 85 patients experienced a total of 12 infections, of which four were reported as mild, four as moderate and four as severe. Nine infections were identified to be of bacterial, two of viral and one of fungal origin. Among the bacterial infections were reports of Listeria meningitis, cellulitis, pseudomembranous colitis and Salmonella infection. One of the viral infections was classified as herpes labialis, the fungal infection was a candida esophagitis. Eight of the 12 reported infections led to hospitalization and four of these required i.v. antibiotic treatment (Tables 4 and 5). One patient with a diagnosed antiphospholipid syndrome experienced a deep vein thrombosis. No other relevant AEs and no deaths occurred during the treatment period and within six months after the last infusion. 
Table 3 Effect of rituximab treatment on selected clinical and laboratory parameters of SLE activity; means with standard deviation for SELENA-SLEDAI

\begin{tabular}{llll}
\hline & First infusion & Last control & $\mathrm{p}$ \\
\hline SELENA-SLEDAI & $12.2 \pm 7.4$ & $3.3 \pm 3.1$ & $<0.05$ \\
Leucopenia (\%) & 20.5 & 9.1 & $<0.05$ \\
Elevated ESR (\%) & 64.6 & 60.9 & 0.72 \\
Low C3 (\%) & 68.1 & 33.3 & $<0.05$ \\
Low C4 (\%) & 62.7 & 43.8 & $<0.05$ \\
Elevated dsDNA antibodies (\%) & 73.4 & 62.2 & 0.29 \\
Any proteinuria (\%) & 38.8 & 39.2 & 0.98 \\
Proteinuria $>500 \mathrm{mg} / 24$ hours (\%) & 27.1 & 16.5 & 0.13 \\
\hline
\end{tabular}

SLE: systemic lupus erythematosus; SELENA-SLEDAI: Safety of Estrogens in Lupus Erythematosus National Assessment-Systemic Lupus Erythematosus Disease Activity Index; ESR: erythrocyte sedimentation rate; dsDNA: double-stranded DNA. Reference values: leucocytes: $\quad 4.0-11.0 \times 10^{3} / \mu \mathrm{l}, \quad \mathrm{ESR}:<20 \mathrm{~mm} / \mathrm{h} \quad($ male),$<30 \mathrm{~mm} / \mathrm{h}$ (female), C3: $90-180 \mathrm{mg} / \mathrm{dl}, \mathrm{C} 4: 10-40 \mathrm{mg} / \mathrm{dl}$, dsDNA antibodies positive according to cut-off values of local laboratories, proteinuria $>150 \mathrm{mg} / 24$ hours.

Table 4 Overview of adverse and serious adverse events

\begin{tabular}{ll}
\hline & $\mathrm{n}(\%)$ \\
\hline Infusion related $^{\mathbf{a}}$ & \\
Infusion reactions $^{\text {Allergic reactions }}$ & $6(7.1)$ \\
Other reaction & $5(5.9)$ \\
Reaction leading to permanent discontinuation & $7(8.2)$ \\
Clinically relevant infections & \\
None & $1(1.2)$ \\
Mild & $73(85.9)$ \\
Moderate & $4(4.7)$ \\
Severe & $4(4.7)$ \\
Observation period (patient years) & $4(4.7)$ \\
Infections per 100 patient years & 66.8 \\
Severe infections per 100 patient years & 19.5 \\
Deaths & 6.0 \\
\hline
\end{tabular}

${ }^{\text {a }}$ During or up to 24 hours after infusion; ${ }^{b} \mathrm{Up}$ to six months after infusion.

\section{Discussion}

The subgroup of SLE patients represents the largest within the GRAID registry, which was initiated to document safety and efficacy of rituximab off-label use in different autoimmune diseases. Data on rituximab use in the SLE population have become even more important, since two randomized controlled trials failed to prove the efficacy of the drug in SLE and lupus nephritis. ${ }^{10,11}$ Concerns regarding the design of these trials became apparent immediately after their publication, as a substantial number of reports and personal experience of many experts in the field has seen a quite convincing effect of the drug in SLE. ${ }^{12,13}$ Further evidence for the usefulness of B-cell targeting therapies comes from the recently approved belimumab, which blocks the B-lymphocyte stimulator BLyS and which has shown efficacy in treating refractory SLE. ${ }^{22-24}$

Despite the retrospective nature of GRAID, we present data from a representative SLE cohort. As can be expected in the off-label setting of rituximab treatment in SLE, the cohort features comparably active and severe disease as indicated by a baseline mean SELENA-SLEDAI of 12.2 and $36.7 \%$ of patients with biopsy-proven lupus nephritis. Nonetheless, a structured efficacy assessment as usually adopted in a prospective clinical trial was not possible. In addition, the heterogeneity of the disease further complicates the assessment of disease activity. Further, assessment tools like the SELENA-SLEDAI or the British Isles Lupus Activity Group (BILAG) score, which have been developed to cover the multiple disease manifestations, are time consuming and less feasible for daily clinical practice. ${ }^{25}$ For these reasons, the

Table 5 Overview of characteristics and outcome of reported infections

\begin{tabular}{|c|c|c|c|c|c|}
\hline No. & Diagnosis & Grade & Hospitalization & I.v. antibiotic & Outcome \\
\hline 1 & Pseudomembranous colitis & Severe & Yes & No & Resolved \\
\hline 2 & Bacterial meningitis & Severe & Yes & Yes & Resolved \\
\hline 3 & Cellulitis of the hand & Severe & Yes & Yes & Resolved \\
\hline 4 & Meningitis (L. monocytogenes) & Severe & Yes & Yes & Resolved \\
\hline 5 & Viral infection $^{\mathrm{a}}$ & Moderate & Yes & Yes & Resolved \\
\hline 6 & Tracheobronchitis & Moderate & Yes & No & Resolved \\
\hline 7 & Not specified & Moderate & Not specified & Not specified & Resolved \\
\hline 8 & Soor esophagitis & Moderate & Yes & No & Resolved \\
\hline 9 & Bacterial infection $^{\mathrm{a}}$ & Mild & No & No & Resolved \\
\hline 10 & Herpes labialis & Mild & No & No & Resolved \\
\hline 11 & Urinary tract infection (E. coli) & Mild & No & No & Resolved \\
\hline 12 & Bacterial infection $^{\mathrm{a}}$ & Mild & No & No & Resolved \\
\hline
\end{tabular}

I.v.: intravenous. ${ }^{\text {a }}$ Not further specified. 
general clinical impression of the treating physician, taking into account the patient history and the laboratory values, is likely to be a good alternative to assess the treatment response in the setting of our study.

Taking this as a key parameter, rituximab treatment showed excellent efficacy in the GRAID SLE population (Figure 1). A possible bias may have been introduced by the non-blinded fashion of the study and the fact that the physician prescribing an off-label medication is convinced of its usefulness. However, in the light of the impressive treatment responses across many disease features, it is unlikely that this bias spoils the overall conclusions. In regard to the effect of specific disease features, general disease manifestations as well as dermatologic and haematologic manifestations showed best, i.e. significant improvements with treatment of rituximab, while manifestations such as musculoskeletal disease manifestations showed a trend towards improvement that did not reach statistical significance (Figure 2). In addition, SELENA-SLEDAI scores as well as laboratory markers of disease activity such as the proportion of patients with leucopenia or abnormal complement levels improved significantly while others showed a tendency for improvement, e.g. the proportion of dsDNA antibody positivity (Table 3 ). These effects on SLE manifestations are consistent with previously published reports on the use of rituximab in SLE. $8,26,27$

A strength of data from a real-life setting in contrast to a clinical trial is that patients can be included who otherwise would not qualify for a clinical trial because of concomitant diseases or treatments. This is of particular importance for the safety assessment of a drug. In this regard, our data on safety show comparable results to those published for RA and SLE clinical trials and post-approval registries. ${ }^{28,29}$ Infusion-related reactions have been reported quite frequently but have rarely led to treatment discontinuation (Table 4). The slightly higher rates of overall and of severe infections may generate from the higher disease burden of SLE and the frequent use of more aggressive immunosuppressants than in an RA population. $^{30,31}$ For example, all patients in the cohort had a pretreatment with corticosteroids and one-third of them with cyclophosphamide. Therefore, a rate of six severe infections per 100 patient years does not seem unexpected (Table 5). The kind of infections is also similar to the experience in RA patients. ${ }^{32,33}$

This cohort adds to registry data from other countries, which have all shown a substantial effect of rituximab in the treatment of SLE. ${ }^{12,34,35}$ The French Autoimmunity and Rituximab (AIR) registry, for example, investigated the efficacy of rituximab in 136 SLE patients, of whom 40 patients had biopsy-proven lupus nephritis. In regard to the general baseline characteristics and the assessment of efficacy, both cohorts are comparable. The overall response rates as estimated by the treating physicians were similar with $83 \%$ in the French and $81 \%$ in our cohort. Although not directly comparable, SELENA-SLEDAI responses as well as manifestation-specific responses were also similar. Concerning safety issues, two severe infusion reactions and five serum-sickness-like reactions occurred in AIR. Twelve (9\%) patients had severe infections, of whom five died because of infection. Because of AEs, rituximab had to be discontinued in 12 patients in AIR. In addition, the Spanish LESIMAB registry evaluated rituximab treatment in 116 SLE patients. Complete response was defined as a SELENA-SLEDAI score of $\leq 2$ with a SELENA-SLEDAI flare index of 0 ; partial response was defined as a reduction in the SELENA-SLEDAI score of $\geq 4$ with no new or worsening of symptoms. According to that, $62.9 \%$ achieved an overall response by month 6 . The median time to response was 6.5 months. A total of $38.1 \%$ of the patients relapsed after the first infusion. Of note, their rate of serious infections was 12.6 per 100 patient years compared to 6.0 per 100 patient years in the GRAID registry.

Together with these data from other European registries, our results show that the off-label use of rituximab in treatment-refractory SLE is common, efficacious and seems to have an acceptable risk profile.

\section{Funding}

This work was supported in part by an unrestricted grant from Roche Pharma AG Germany without involvement in the study design or the collection, analysis or interpretation of the data.

\section{Conflicts of interest}

M. Witt, M. Grunke, M. Baeuerle, M. Aringer, G. R. Burmester, M. Fleck, M. Haubitz, A. RubbertRoth, H. Tony, T. Dörner and H. Schulze-Koops have received speaker fees from Roche. M. Aringer, M. Haubitz and H. Schulze-Koops have received honoraria as consultants for Roche. T. Dörner has 
received an unrestricted grant for GRAID from Roche. All others have nothing to declare.

\section{Ethical approval}

Ethical approval and approval by the local data protection agency were obtained by Charité Universitaetsmedizin Berlin, Germany. Local approvals were obtained by the principal investigators if required by local regulations.

\section{References}

1 Edwards JC, Cambridge G. B-cell targeting in rheumatoid arthritis and other autoimmune diseases. Nat Rev Immunol 2006; 6: 394-403.

2 Dörner T, Radbruch A, Burmester GR. B-cell-directed therapies for autoimmune disease. Nat Rev Rheumatol 2009; 5: 433-441.

3 Zandman-Goddard G, Orbach H, Shoenfeld Y. Novel approaches to therapy for systemic lupus erythematosus: Update 2005. Expert Rev Clin Immunol 2005; 1: 223-238.

4 Dörner T, Isenberg D, Jayne D, Wiendl H, Zillikens D, Burmester G. Current status on B-cell depletion therapy in autoimmune diseases other than rheumatoid arthritis. Autoimmun Rev 2009; 9: $82-89$.

5 Stone JH, Merkel PA, Spiera R, et al. Rituximab versus cyclophosphamide for ANCA-associated vasculitis. $N$ Engl J Med 2010; 363: 221-232.

6 Jones RB, Tervaert JW, Hauser T, et al. Rituximab versus cyclophosphamide in ANCA-associated renal vasculitis. $N$ Engl $J$ Med 2010; 363: 211-220.

7 Leandro MJ, Edwards JC, Cambridge G, Ehrenstein MR, Isenberg DA. An open study of B lymphocyte depletion in systemic lupus erythematosus. Arthritis Rheum 2002; 46: 2673-2677.

$8 \mathrm{Lu}$ TY, Ng KP, Cambridge G, et al. A retrospective seven-year analysis of the use of $\mathrm{B}$ cell depletion therapy in systemic lupus erythematosus at University College London Hospital: The first fifty patients. Arthritis Rheum 2009; 61: 482-487.

9 Isenberg DA. Rituximab - it was the best of times, it was the worst of times. Autoimmun Rev 2012; 11: 790-791.

10 Merrill J, Buyon J, Furie R, et al. Assessment of flares in lupus patients enrolled in a phase II/III study of rituximab (EXPLORER). Lupus 2011; 20: 709-716.

11 Rovin BH, Furie R, Latinis K, et al. Efficacy and safety of rituximab in patients with active proliferative lupus nephritis: The Lupus Nephritis Assessment with Rituximab study. Arthritis Rheum 2012; 64: 1215-1226.

12 Fernández-Nebro A, de la Fuente JM, Carreño L, et al. Multicenter longitudinal study of B-lymphocyte depletion in refractory systemic lupus erythematosus: The LESIMAB study. Lupus 2012; 21: 1063-1076.

13 Weidenbusch M, Rommele C, Schrottle A, Anders HJ. Beyond the LUNAR trial. Efficacy of rituximab in refractory lupus nephritis. Nephrol Dial Transplant 2013; 28: 106-111.

14 Díaz-Lagares C, Croca S, Sangle S, et al. Efficacy of rituximab in 164 patients with biopsy-proven lupus nephritis: Pooled data from European cohorts. Autoimmun Rev 2012; 11: 357-364.

15 Andrade-Ortega L, Irazoque-Palazuelos F, López-Villanueva R, et al. Efficacy of rituximab versus cyclophosphamide in lupus patients with severe manifestations. A randomized and multicenter study [in Spanish]. Reumatol Clin 2010; 6: 250-255.

16 Turner-Stokes T, Lu TY, Ehrenstein MR, Giles I, Rahman A, Isenberg DA. The efficacy of repeated treatment with B-cell depletion therapy in systemic lupus erythematosus: An evaluation. Rheumatology (Oxford) 2011; 50: 1401-1408.

17 van Vollenhoven RF. Rituximab - shadow, illusion or light? Autoimmun Rev 2012; 11: 563-567.

18 Tony HP, Burmester G, Schulze-Koops H, et al. Safety and clinical outcomes of rituximab therapy in patients with different autoimmune diseases: Experience from a national registry (GRAID). Arthritis Res Ther 2011; 13: R75.

19 Hochberg MC. Updating the American College of Rheumatology revised criteria for the classification of systemic lupus erythematosus. Arthritis Rheum 1997; 40: 1725.

20 Bombardier C, Gladman DD, Urowitz MB, Caron D, Chang CH. Derivation of the SLEDAI. A disease activity index for lupus patients. The Committee on Prognosis Studies in SLE. Arthritis Rheum 1992; 35: 630-640.

21 Trotti A, Colevas AD, Setser A, et al. CTCAE v3.0: Development of a comprehensive grading system for the adverse effects of cancer treatment. Semin Radiat Oncol 2003; 13: 176-181.

22 Navarra SV, Guzmán RM, Gallacher AE, et al. Efficacy and safety of belimumab in patients with active systemic lupus erythematosus: A randomised, placebo-controlled, phase 3 trial. Lancet 2011; 377: 721-731.

23 Furie R, Petri M, Zamani O, et al. A phase III, randomized, placebo-controlled study of belimumab, a monoclonal antibody that inhibits B lymphocyte stimulator, in patients with systemic lupus erythematosus. Arthritis Rheum 2011; 63: 3918-3930.

24 Manzi S, Sánchez-Guerrero J, Merrill JT, et al. Effects of belimumab, a B lymphocyte stimulator-specific inhibitor, on disease activity across multiple organ domains in patients with systemic lupus erythematosus: Combined results from two phase III trials. Ann Rheum Dis 2012; 71: 1833-1838.

25 Hay EM, Bacon PA, Gordon C, et al. The BILAG index: A reliable and valid instrument for measuring clinical disease activity in systemic lupus erythematosus. $Q J$ Med 1993; 86: 447-458.

26 Bang SY, Lee CK, Kang YM, et al. Multicenter retrospective analysis of the effectiveness and safety of rituximab in Korean patients with refractory systemic lupus erythematosus. Autoimmune Dis 2012; 2012: 565039

27 Jonsdottir T, Zickert A, Sundelin B, Henriksson EW, van Vollenhoven RF, Gunnarsson I. Long-term follow-up in lupus nephritis patients treated with rituximab - clinical and histopathological response. Rheumatology (Oxford) 2013; 52: 847-855.

28 Isvy A, Meunier M, Gobeaux-Chenevier C, et al. Safety of rituximab in rheumatoid arthritis: A long-term prospective single-center study of gammaglobulin concentrations and infections. Joint Bone Spine 2012; 79: 365-369.

29 Curtis JR, Xie F, Chen L, et al. The comparative risk of serious infections among rheumatoid arthritis patients starting or switching biological agents. Ann Rheum Dis 2011; 70: 1401-1406.

30 Yildirim-Toruner C, Diamond B. Current and novel therapeutics in the treatment of systemic lupus erythematosus. J Allergy Clin Immunol 2011; 127: 303-312; quiz 13-14.

31 Gough AK, Foo J. Current treatment of rheumatoid arthritis. BMJ 2011; 343: d7050; author reply d2.

32 van Vollenhoven RF, Emery P, Bingham 3rd CO, et al. Longterm safety of patients receiving rituximab in rheumatoid arthritis clinical trials. J Rheumatol 2010; 37: 558-567.

33 Gottenberg JE, Ravaud P, Bardin T, et al. Risk factors for severe infections in patients with rheumatoid arthritis treated with rituximab in the autoimmunity and rituximab registry. Arthritis Rheum 2010; 62: 2625-2632.

34 Terrier B, Amoura Z, Ravaud P, et al. Safety and efficacy of rituximab in systemic lupus erythematosus: Results from 136 patients from the French AutoImmunity and Rituximab registry. Arthritis Rheum 2010; 62: 2458-2466.

35 Ramos-Casals M, Brito-Zerón P, Muñoz S, Soto MJ. A systematic review of the off-label use of biological therapies in systemic autoimmune diseases. Medicine (Baltimore) 2008; 87: 345-364. 